\title{
On an Extremal Problem for Poset Dimension
}

\author{
Grzegorz Guśpiel $^{1}$ (D) P Piotr Micek ${ }^{1}$ • Adam Polak ${ }^{1}$
}

Received: 22 May 2017 / Accepted: 1 November 2017 / Published online: 22 November 2017

(C) The Author(s) 2017. This article is an open access publication

\begin{abstract}
Let $f(n)$ be the largest integer such that every poset on $n$ elements has a 2dimensional subposet on $f(n)$ elements. What is the asymptotics of $f(n)$ ? It is easy to see that $f(n)>=n^{1 / 2}$. We improve the best known upper bound and show $f(n)=\mathcal{O}\left(n^{2 / 3}\right)$. For higher dimensions, we show $f_{d}(n)=\mathcal{O}\left(n^{\frac{d}{d+1}}\right)$, where $f_{d}(n)$ is the largest integer such that every poset on $n$ elements has a $d$-dimensional subposet on $f_{d}(n)$ elements.
\end{abstract}

Keywords Partially ordered sets · Poset dimension · Extremal combinatorics · Permutation matrices

\section{Introduction}

Every partially ordered set on $n$ elements has a chain or an antichain of size at least $n^{1 / 2}$, this is an immediate consequence of Dilworth's Theorem or its easier dual counterpart. Chains and antichains are very special instances of 2-dimensional posets. Surprisingly, the following simple problem is open:

Grzegorz Guśpiel was partially supported by the Polish Ministry of Science and Higher Education grant DI2013 000443. Piotr Micek was partially supported by the National Science Center of Poland under grant no. 2015/18/E/ST6/00299. Adam Polak was partially supported by the Polish Ministry of Science and Higher Education program "Diamentowy Grant".

Grzegorz Guśpiel guspiel@tcs.uj.edu.pl

Piotr Micek

micek@tcs.uj.edu.pl

Adam Polak

polak@tcs.uj.edu.pl

1 Theoretical Computer Science Department, Faculty of Mathematics and Computer Science, Jagiellonian University, Kraków, Poland 
Let $f(n)$ be the largest integer such that every poset on $n$ elements has a 2-dimensional subposet on $f(n)$ elements. What is the asymptotics of $f(n)$ ?

Although this sounds like a natural extremal-type question for posets, it was posed only in 2010, by François Dorais [1]. Clearly, $n^{1 / 2} \leqslant f(n) \leqslant n$. Reiniger and Yeager [5] proved a sublinear upper bound, that is $f(n)=\mathcal{O}\left(n^{0.8295}\right)$. Their construction is a lexicographic power of standard examples.

The main idea behind our contribution was a belief that a $(k \times k)$-grid is asymptotically the largest 2-dimensional subposet of the $(k \times k \times k)$-cube. This led us to the following theorem:

\section{Theorem 1}

$$
f(n) \leqslant 4 n^{2 / 3}+o\left(n^{2 / 3}\right) .
$$

Recall that the dimension $\operatorname{dim}(P)$ of a poset $P$ is the least integer $d$ such that elements of $P$ can be embedded into $\mathbb{R}^{d}$ in such a way that $x<y$ in $P$ if and only if the point of $x$ is below the point of $y$ with respect to the product order on $\mathbb{R}^{d}$. Equivalently, the dimension of $P$ is the least $d$ such that there are $d$ linear extensions of $P$ whose intersection is $P$. By convention, whenever we say a poset is $d$-dimensional, we mean its dimension is at most $d$.

Reiniger and Yeager [5] also studied the guaranteed size of the largest $d$-dimensional subposet of poset on $n$ elements. Let $f_{d}(n)$ be the largest integer such that every poset on $n$ elements has a $d$-dimensional subposet on $f_{d}(n)$ elements. They proved, in particular, that $f_{d}(n)=\mathcal{O}\left(n^{g}\right)$, where $g=\log _{2 d+2}(2 d+1)$.

Let $[n]$ denote $\{0,1, \ldots, n-1\}$. By the $\mathbf{n}^{\mathbf{d}}$-grid we mean the poset on the ground set $[n]^{d}$ with the natural product order, i.e. $\left(x_{1}, x_{2}, \ldots, x_{d}\right) \leqslant\left(y_{1}, y_{2}, \ldots, y_{d}\right)$ if $x_{i} \leqslant y_{i}$ for all $i$. Note that the $\mathbf{n}^{\mathbf{d}}$-grid is a $d$-dimensional poset. Moreover, it is easy to see that the $\mathbf{n}^{\mathbf{d}+\mathbf{1}}$ grid contains as a subposet the $\mathbf{n}^{\mathbf{d}}$-grid - simply fix one coordinate to an arbitrary value. We prove that this is asymptotically the largest $d$-dimensional subposet of the $\mathbf{n}^{\mathbf{d}+\mathbf{1}}$-grid. For $d \leqslant 7$, this observation improves on the best known upper bound for the asymptotics of $f_{d}(n)$.

\section{Theorem 2}

$$
f_{d}(n)=\mathcal{O}\left(n^{\frac{d}{d+1}}\right)
$$

In order to show this we apply a multidimensional version of the theorem by Marcus and Tardos [3] saying that the number of 1-entries in an $n \times n(0,1)$-matrix that avoids a fixed permutation matrix $P$ is $\mathcal{O}(n)$. The multidimensional version was proved by Klazar and Marcus [2], and then independently by Methuku and Pálvölgyi [4], who applied it to another extremal problem related to subposets, i.e. they proved that for every poset $P$ the size of any family of subsets of $[n]$ that does not contain $P$ as a subposet is at most $\mathcal{O}\left(\left(\begin{array}{c}n \\ \lfloor n / 2\rfloor\end{array}\right)\right)$.

\section{Dimension Two}

If we ignore a multiplicative constant, Theorem 1 becomes a special case of Theorem 2 . Still, we provide a short and simple proof of Theorem 1, as we believe it might provide a better insight to the core of the problem. 

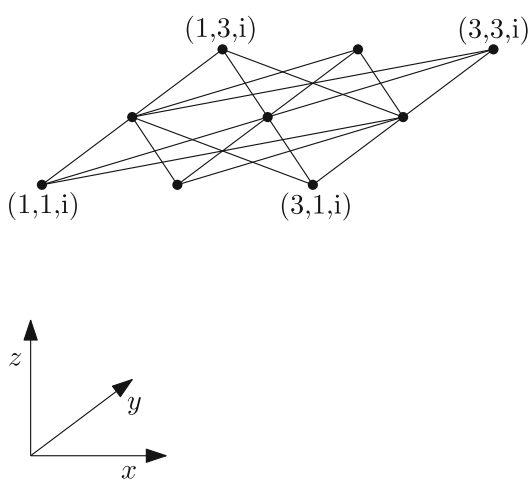

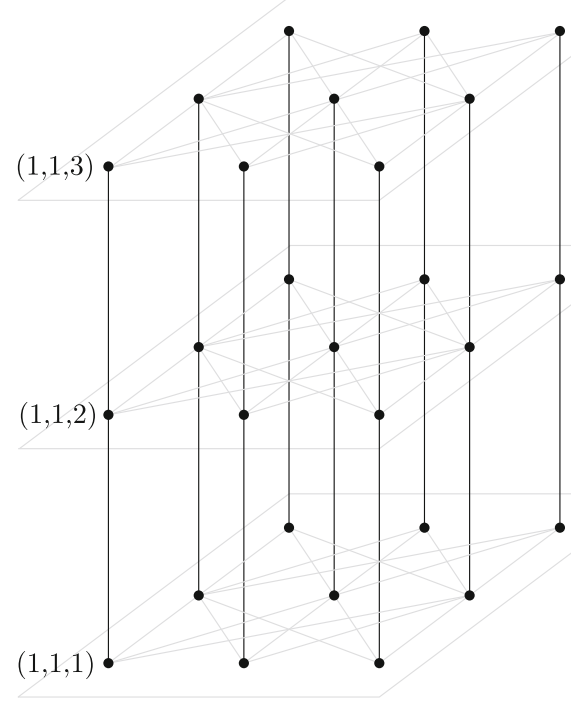

Fig. 1 A subposet of $\mathbf{C}_{\mathbf{3}}$ composed of all elements with $z$-coordinate equal to $i$ (on the left) and the poset $\mathbf{C}_{\mathbf{3}}$ itself (on the right)

Proof of Theorem 1 First we argue for values of $n$ such that $n=r^{3}$ for some $r \in \mathbb{N}$. Then at the end of the proof we address the general case.

Let $\mathbf{C}_{\mathbf{r}}$ be the poset with the ground set $[r]^{3}$, where $\left(x_{1}, y_{1}, z_{1}\right) \leqslant \mathbf{C}_{\mathbf{r}}\left(x_{2}, y_{2}, z_{2}\right)$ if

$$
\left(z_{1} \leqslant z_{2}\right) \text { and }\left(y_{1}<y_{2} \text { or }\left(y_{1}=y_{2} \text { and } x_{1}=x_{2}\right)\right) \text {, }
$$

see Fig. 1.

Consider any subposet $S$ of $\mathbf{C}_{\mathbf{r}}$ such that $|S| \geqslant 4 r^{2}$. We will prove that $\operatorname{dim}(S)>2$ by showing that $S$ contains as a subposet the poset ${ }^{1}$ of dimension 3 presented in Fig. 2.

Let $S^{1}$ be the poset obtained from $S$ by removing every element $(x, y, z)$ such that $S$ contains no element $\left(x, y, z^{\prime}\right)$ with $z^{\prime}<z$. Note that $\left|S^{1}\right| \geqslant 3 r^{2}$, as for every pair $(x, y) \in$ $[r]^{2}$ at most one element is removed. Now by the pigeonhole principle, we get that $S^{1}$ contains a subposet $S^{2}$ on at least $3 r$ elements such that all elements of $S^{2}$ have the same $z$-coordinate.

Let $A$ be any point in $S^{2}$ with the minimal $y$-coordinate and let $S^{3}$ be the subposet of $S^{2}$ obtained by removing all points with the same $y$-coordinate as $A$. As there can be at most $r$ points with the same $y$-coordinate, $\left|S^{3}\right| \geqslant 2 r$. By the pigeonhole principle for $r-1$ containers, $S^{3}$ contains three points with the same $y$-coordinate, say $B_{1}=\left(x_{1}, y, z\right)$, $B_{2}=\left(x_{2}, y, z\right), B_{3}=\left(x_{3}, y, z\right)$. Thanks to the removal rule that led to the creation of $S^{1}$, the poset $S$ contains points $C_{1}=\left(x_{1}, y, z_{1}\right), C_{2}=\left(x_{2}, y, z_{2}\right), C_{3}=\left(x_{3}, y, z_{3}\right)$ for some $z_{1}, z_{2}, z_{3}<z$.

One can easily verify that the subposet $\left\{A, B_{1}, B_{2}, B_{3}, C_{1}, C_{2}, C_{3}\right\}$ of $S$ is the poset in Fig. 2. Since it has dimension 3, we have $\operatorname{dim}(S)>2$, which concludes the proof for $n$ being a perfect cube.

\footnotetext{
${ }^{1}$ This is one of the 3-irreducible posets, which are listed in [6].
} 
Fig. 2 A poset of dimension 3 found in any subposet of $\mathbf{C}_{\mathbf{r}}$ of size at least $4 r^{2}$

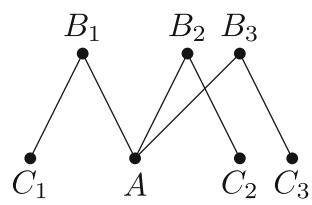

Now, fix any $n \in \mathbb{N}$, and let $r=\lceil\sqrt[3]{n}\rceil$. Note that $f$ is non-decreasing, thus

$$
f(n) \leqslant f\left(r^{3}\right) \leqslant 4 r^{2} \leqslant 4(\sqrt[3]{n}+1)^{2}=4 n^{2 / 3}+o\left(n^{2 / 3}\right) .
$$

With a more tedious analysis, which involves one more forbidden subposet and removal of both lowest and highest $z$-coordinate points in each $(x, y)$-column, we can prove a slightly stronger upper bound, i.e. $f(n) \leqslant 3 n^{2 / 3}+o\left(n^{2 / 3}\right)$. However, we do not know how to improve on the asymptotics of $f$.

\section{Higher Dimensions}

In this section we prove Theorem 2. In order to do this we apply a multidimensional version of the theorem by Marcus and Tardos [3], proved by Klazar and Marcus [2]. First, we recall their result. The original terminology can be simplified because our argument does not use arbitrary sized matrices and we can focus only on multidimensional analogs of square matrices.

We call a subset of $[n]^{d}$ a d-dimensional $(0,1)$-matrix.

For two $d$-dimensional $(0,1)$-matrices $A \subseteq[n]^{d}$ and $B \subseteq[k]^{d}$, we say that $A$ contains $B$ if there exist $d$ increasing injections $h_{i}:[k] \rightarrow[n], i \in\{1,2, \ldots, d\}$, such that

$$
\text { if }\left(x_{1}, x_{2}, \ldots, x_{d}\right) \in B \text {, then }\left(h_{1}\left(x_{1}\right), h_{2}\left(x_{2}\right), \ldots, h_{d}\left(x_{d}\right)\right) \in A \text {, }
$$

for all $\left(x_{1}, x_{2}, \ldots, x_{d}\right) \in[k]^{d}$. Otherwise, we say that $A$ avoids $B$.

We say that $A \subseteq[n]^{d}$ is a d-dimensional permutation of $[n]$

$$
|A|=n \quad \text { and } \quad \forall_{\substack{x, y \in A \\ x \neq y}} \forall_{i \in\{1,2, \ldots, d\}} x_{i} \neq y_{i} .
$$

In other words, the size of the projection of $A$ onto the $i$-th dimension equals $n$ for each $i \in\{1,2, \ldots, d\}$.

Theorem 3 (Klazar-Marcus [2]) For every fixed d-dimensional permutation $P$ the maximum number of elements of a d-dimensional matrix $A \subseteq[n]^{d}$ that avoids $P$ is $\mathcal{O}\left(n^{d-1}\right)$.

Now we are ready to prove the following statement, which clearly implies Theorem 2.

Theorem 4 The largest d-dimensional subposet of the $\mathbf{n}^{\mathbf{d}+\mathbf{1}}$-grid has $\mathcal{O}\left(n^{d}\right)$ elements.

Proof We fix any poset of dimension $d+1$, e.g. the standard example $S_{d+1}$, i.e. the inclusion order of singletons and $d$-element subsets of $[d+1]$. Now, we fix a realizer of $S_{d+1}$ of size 
$d+1$, i.e. a set of $d+1$ linear orders $\left\{L_{1}, L_{2}, \ldots, L_{d+1}\right\}$ such that $L_{1} \cap L_{2} \cap \cdots \cap L_{d+1}=$ $S_{d+1}$. Finally, we construct a $(d+1)$-dimensional permutation $P \subseteq[2(d+1)]^{d+1}$ such that $\left(x_{1}, x_{2}, \ldots, x_{d+1}\right) \in P$ if and only if there exists $x \in S_{d+1}$ such that $x$ is the $x_{i}$-th element of $L_{i}$ for each $i \in\{1,2, \ldots, d+1\}$. Note that the natural product order of elements of $P$ is isomorphic to $S_{d+1}$.

Now, take any $d$-dimensional subposet of the $\mathbf{n}^{\mathbf{d}+\mathbf{1}}$-grid and denote by $A$ the set of its elements. In particular, the subposet does not contain $S_{d+1}$ as a subposet. Note that it implies that $A$ avoids $P$, thus by Theorem 3 the size of the subposet is $\mathcal{O}\left(n^{d}\right)$.

Note that the proof above does not exploit any specific properties of the standard example, apart from its dimension. In particular, it implies that every $(d+1)$-dimensional poset

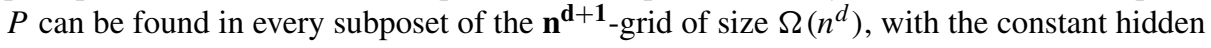
in the asymptotic notation depending on the choice of $P$.

Acknowledgements We send thanks to Wojciech Samotij and Dömötör Pálvölgyi for pointing us to useful references.

Open Access This article is distributed under the terms of the Creative Commons Attribution 4.0 International License (http://creativecommons.org/licenses/by/4.0/), which permits unrestricted use, distribution, and reproduction in any medium, provided you give appropriate credit to the original author(s) and the source, provide a link to the Creative Commons license, and indicate if changes were made.

\section{References}

1. Dorais, F.G.: Subposets of small Dushnik-Miller dimension. MathOverflow. http://mathoverflow.net/ questions/29169 (2010). Accessed 13 Feb 2016

2. Klazar, M., Marcus, A.: Extensions of the linear bound in the Füredi-Hajnal conjecture. Adv. Appl. Math. 38(2), 258-266 (2007). https://doi.org/10.1016/j.aam.2006.05.002

3. Marcus, A., Tardos, G.: Excluded permutation matrices and the Stanley-Wilf conjecture. J. Comb. Theory A 107(1), 153-160 (2004). https://doi.org/10.1016/j.jcta.2004.04.002

4. Methuku, A., Pálvölgyi, D.: Forbidden hypermatrices imply general bounds on induced forbidden subposet problems. Combin. Probab. Comput. 26(4), 593-602 (2017). https://doi.org/10.1017/S0963548317 000013

5. Reiniger, B., Yeager, E.: Large subposets with small dimension. Order 33(1), 81-84 (2016). https://doi.org/ 10.1007/s11083-015-9353-0

6. Trotter, W.T.: Combinatorics and Partially Ordered Sets: Dimension Theory. Johns Hopkins University Press, Baltimore (1992) 